\title{
Dynamic Spectrum Management in Green Cognitive Radio Cellular Networks
}

\author{
Lokman Sboui $^{1}$, Hakim Ghazzai ${ }^{2}$, Zouheir Rezki ${ }^{3}$, and Mohamed-Slim Alouini ${ }^{1}$ \\ ${ }^{1}$ King Abdullah University of Science and Technology (KAUST), Thuwal, Makkah Province, Saudi Arabia, \\ \{lokman.sboui, slim.alouini\}@kaust.edu.sa \\ ${ }^{2}$ Qatar Mobility Innovations Center (QMIC), Qatar University, Doha, Qatar, hakimg@qmic.com \\ ${ }^{3}$ University of Idaho, Moscow, ID, USA, zrezki@uidaho.edu
}

\begin{abstract}
In this paper, we propose a new cellular network operation scheme fulfilling the $5 \mathrm{G}$ requirements related to spectrum management and green communications. We focus on cognitive radio cellular networks in which both the primary network (PN) and the secondary network (SN) are maximizing their operational profits. The $\mathrm{PN}$ and the $\mathrm{SN}$ are required to respect a $\mathrm{CO}_{2}$ emissions threshold by switching off one or more lightly loaded base stations (BSs). In addition, the PN accepts to cooperate with the $\mathrm{SN}$ by leasing its spectrum in the cells where the PN is turned off. In return, the corresponding SN BSs host the PN users and impose extra roaming fees to the PN. We propose a low-complexity algorithm that maximizes the profit per $\mathrm{CO}_{2}$ emissions metric while switching on/off the BSs. In the simulations, we show that our proposed algorithm achieves performances close to the exhaustive search method. In addition, we find that the roaming price is a key parameter that affects both $\mathrm{PN}$ and $\mathrm{SN}$ profits ${ }^{1}$.

Index Terms-Collaborative cellular networks, spectrum cooperation, green cognitive radio networking.
\end{abstract}

\section{INTRODUCTION}

In cellular networks, the energy consumption of the base stations (BSs) is around $60 \%$ of the total network consumption [2]. Hence, the main focus of the operators' is to reduce their expenses by reducing the BS consumption and, therefore, maximizing their profit. As a result, reducing the energy consumption is an obligation due to the heavy energy bills [3] and the high $\mathrm{CO}_{2}$ footprint of the cellular networks which present $0.2 \%$ of the global emissions [4]. Also, in the next generation cellular network design, the energy-efficiency and environmentawareness as well as dynamic spectrum management are required to be jointly considered [5]. Previous works studied the green collaboration between the primary network (PN) and the secondary network (SN) in the CR cellular networks [6]-[10]. In [8], the SN maximizes its achievable rate while the PN is minimizing its energy consumption. In [10], the two operators collaborate

\footnotetext{
${ }^{1}$ Extension of this work has been published in IEEE Access [1].

978-1-5386-3531-5/17/\$31.00 (C) 2017 IEEE
}

while minimizing the fossil fuel cost. Although the aim of these studies is to optimize the green collaboration between both networks, the microgrid aspects with dynamic pricing was not considered. In addition, the $\mathrm{CO}_{2}$ emissions are not considered as the main metric for green communications in the cellular networks.

To address these challenges, we propose, in this paper, an energy-aware and spectrum-aware operation for CR cellular networks. In addition, the BSs are powered by a microgrid that includes renewable energy. Hence, the dynamic energy pricing at the microgrid levels and the renewable energy availability are considered as constraints of the profit maximization problem. The PN reduces its $\mathrm{CO}_{2}$ footprint and saves energy by cooperating with the $\mathrm{SN}$. We formulate a profit maximization problem while respecting a $\mathrm{CO}_{2}$ emissions threshold and maintaining a required level of quality of service (QoS). The $\mathrm{CO}_{2}$ emissions reduction is performed by switching off some of the PN BSs while offloading the corresponding users to the closest SN's BSs. In exchange, the SN fully uses the corresponding bandwidth and receives a roaming reward from the PN. We propose a low-complexity algorithm to switch on/off the BSs based on a profit per $\mathrm{CO}_{2}$ emissions metric.

The main contributions of this work are:

- Developing a green model for collaborative CR cellular networks aiming to maximize both profits,

- Proposing a low-complexity algorithm managing BS on/off switching, roaming, and spectrum,

- Comparing our proposed algorithm's performance to that of the exhaustive search method.

The rest of this paper, we present the system model in Section II. In Section III, the problem formulation is presented. In Section IV, the proposed algorithm for $\mathrm{PN}$ and $\mathrm{SN}$ is developed. Numerical results are given in Section V. Finally, the paper is concluded in Section VI. 


\section{SySTEM MODEL}

\section{A. Cellular Network Model}

We consider two mobile operators operating over the same frequency bandwidth and same area: an operator licensed to use the frequency bandwidth denoted by $W$, i.e., the PN, and an operator that shares the bandwidth, i.e., the SN, in exchange of eventual users roaming from the PN. The PN and the SN serve $N_{p}$ and $N_{s}$ users using $N_{\text {BS }}$ BSs modeled as hexagonal cells each, respectively. The SN BSs are deployed on the PN cells edge to avoid the interferences and to cover the same overall area as shown in Fig. 1.

Each PN and SN user is exploiting a certain service provided by the operator and denoted by $\Sigma_{p}$ and $\Sigma_{s}$, respectively. The number of users of network $i$ is denoted by $N_{i, j}^{(\sigma)}$, where $i \in\{p, s\}$ denotes the PN and $\mathrm{SN}$, respectively. In addition, the type of provided service is denoted by $\sigma, \sigma=1, \cdots, \Sigma_{i}$. We denote by $f_{i, \sigma}\left(x_{i}, y_{i}\right)$ the joint probability density function of the coordinates of the users of a network $i$ exploiting a service $\sigma$. Also, we denote by $P_{i, j}$ the power consumed at the $j$-th BS at the network $i$ which is derived as [11]:

$$
P_{i, j}=a_{i} \sum_{\sigma=1}^{\Sigma_{i}} \sum_{k=1}^{N_{i, j}^{(\sigma)}} p_{i, j}^{(k, \sigma)}+b_{i},
$$

where $p_{i, j}^{(k, \sigma)}$ is the transmission power of the $k$-th user exploiting the service $\sigma$ in the $j$-th BS, $a_{i}$ is a coefficient related to both amplification and feeder losses in the network $i$, and $b_{i}$ is the circuit power that does not dependent on the transmit power [12]. We focus, in this paper, on planning the downlink transmission using average statistics. Hence, we derive the average transmit power per user exploiting the service $\sigma$, denoted by $\bar{p}_{i, j}^{(\sigma)}$. Consequently, eq. (1) becomes $P_{i, j}=$ $a_{i} \sum_{\sigma=1}^{\Sigma_{i}} N_{i, j}^{(\sigma)} \bar{p}_{i, j}^{(\sigma)}+b_{i}$, where $\bar{p}_{i, j}^{(\sigma)}$ is the average power per service $\sigma$ in the $j$-th BS. In addition, for a given service $\sigma$, the average transmit power per user, [11], is $\bar{p}_{i, j}^{(\sigma)}=\frac{P_{\min }^{(\sigma)}}{K} \mathbb{E}_{i, \sigma}\left[d_{i, j}^{\nu}\right]$, where $P_{\min }^{(\sigma)}$ denotes the minimum power needed to any user exploiting the service $\sigma$ to obtain an acceptable QoS. The parameter $K$ represents the effects of the BS's carrier frequency, propagation environment, and antenna settings. The parameter $\nu$ denotes the pathloss exponent and $\mathbb{E}_{i, \sigma}\left[d_{\bar{i}, j}^{\nu}\right]$ is an averaging of the distance function between the $j$-th BS of network $\bar{i}$ and the users of network $i$ exploiting the service $\sigma$ connected to this BS, where $\bar{i}=s$ if $i=p$ and $\bar{i}=p$ if $i=s$. The average distance $\mathbb{E}_{i, \sigma}\left[d_{\bar{i}, j}^{\nu}\right]$ is derived using $C_{i, j}$ defined as the distribution of users exploiting the service $\sigma$ in the $j$-th BS of the network $i$, as:

$\mathbb{E}_{i, \sigma}\left[d_{\bar{i}, j}^{\nu}\right]=\iint_{C_{\bar{i}, j}}\left(\left(u-x_{\bar{i}, j}\right)^{2}+\left(v-y_{\bar{i}, j}\right)^{2}\right)^{\frac{\nu}{2}} f_{i, \sigma}(u, v) d u d v$

where $(u, v)$ are the coordinates of a user exploiting

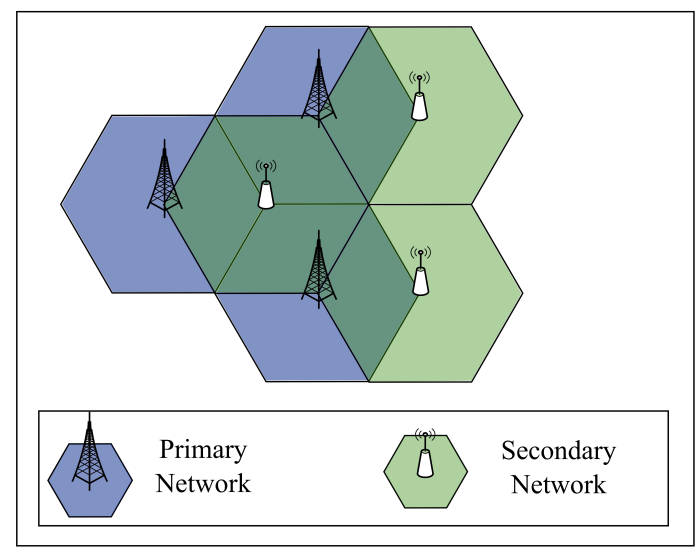

Fig. 1: Primary and secondary cells deployment.

the service $\sigma$ in the network $i$. These coordinates follow the distribution $f_{i, \sigma}(u, v)$. We denote by $\bar{N}_{i}, i \in\{p, s\}$ the maximum number of users that can be served simultaneously by each BS. In our framework, we assume that both networks are well-planned which means that $N_{p, j} \leq \bar{N}_{p}$ and $N_{s, j} \leq \bar{N}_{s}$ where $N_{i, j}$ describes all the users connected to the $j$-th BS in the network $i$ such that $N_{i, j}=\sum_{\sigma=1}^{\Sigma_{i}} N_{i, j}^{(\sigma)}$. However, the number of users served by the SN BSs may exceed the secondary capacity after offloading the PN users. Hence, we define an outage constraint of the PN users. In addition, the QoS in terms of data rate should be higher than a certain minimal required data rate per user exploiting the service $\sigma$ denoted by $r_{i, \sigma}$. Hence, we define the following inequality

$$
w_{i, \sigma} \log _{2}\left(1+\frac{P_{\min }^{(\sigma)}}{w_{i, \sigma} N_{0}}\right) \geq r_{i, \sigma} .
$$

where $N_{0}$ is the noise per bandwidth and $w_{i, \sigma}$ is the bandwidth fraction used by each user exploiting the service $\sigma$ in the network $i$ with a target rate defined in (3). Similarly to [10], $w_{i, \sigma}$ is defined by

$$
w_{i, \sigma}=-\frac{\frac{P_{\min }^{(\sigma)}}{N_{0}}}{1+\frac{P_{\min }^{(\sigma)}}{r_{i, \sigma} N_{0}} \mathcal{W}_{-1}\left(-\frac{r_{i, \sigma} N_{0}}{P_{\min }^{(\sigma)}} e^{-\frac{r_{i, \sigma} N_{0}}{P_{\min }^{(\sigma)}}}\right)}
$$

where $\mathcal{W}_{-1}(\cdot)$ is the lower branch of the W-Lambert function and defined in $\left[-\frac{1}{e}, 0\right]$ [13]. Note that the inequality $\frac{P_{\min }^{(\sigma)}}{r_{i, \sigma} N_{0}} \mathcal{W}_{-1}\left(-\frac{r_{i, \sigma} N_{0}}{P_{\min }^{(\sigma)}} e^{-\frac{r_{i, \sigma} N_{0}}{P_{\min }^{(\sigma)}}}\right)<-1$,is always true. Hence, the bandwidth fraction in (4) is positive.

\section{B. Conditions of Green-Oriented Cellular Networks}

We present four conditions qualifying the PN and SN networks as green-oriented cellular networks: 
1) Limited $\mathrm{CO}_{2}$ footprint: The $\mathrm{CO}_{2}$ emitted by both $\mathrm{PN}$ and $\mathrm{SN}$ is limited by a certain allowed quantity $\overline{\mathrm{CO}_{2}}$ evaluated using a quadratic penalty function [14]. At a BS $j$ of the network $i$, this penalty function, denoted by $\mathcal{F}_{i, j}^{\mathrm{CO}_{2}}$, is defined by [14]:

$\mathcal{F}_{i, j}^{\mathrm{CO}_{2}}=\left\{\begin{array}{cl}0, & \text { if } \mathcal{E}_{i, j} \leq \mathcal{E}_{i, j}^{(g)}, \\ \phi_{i}\left(\mathcal{E}_{i, j}-\mathcal{E}_{i, j}^{(g)}\right)^{2}+\psi_{i}\left(\mathcal{E}_{i, j}-\mathcal{E}_{i, j}^{(g)}\right), & \text { if } \mathcal{E}_{i, j}>\mathcal{E}_{i, j}^{(g)},\end{array}\right.$

where $\phi_{i}$ and $\psi_{i}$ are defined as the pollutant coefficients of the fossil fuel used directly and indirectly to run the BSs of network $i$, and where $\mathcal{E}_{i, j}$ is the energy consumption of the BS $j$ of the network $i$. We denote by $\mathcal{F}_{i}^{\mathrm{CO}_{2}}=\sum_{j} \mathcal{F}_{i, j}^{\mathrm{CO}_{2}}$ the total $\mathrm{CO}_{2}$ emissions of network $i$.

2) BSs Deactivation: Both $\mathrm{PN}$ and $\mathrm{SN}$ are allowed to deactivate one or more BSs to reduce the $\mathrm{CO}_{2}$ footprint. However, if a PN BS is switched off, the corresponding SN BS needs to be active in order to serve the primary roamed users while collecting a roaming reward paid by the PN. We denote by $\epsilon_{i, j}$ the binary variables describing the status of the $j$-th $\mathrm{BS}$ in the network $i$, i.e., $\epsilon_{i, j}=0$ if the BS is switched off, otherwise, $\epsilon_{i, j}=1$. When, $\epsilon_{i, j}=0$ we assume that the corresponding BS is idle with no energy consumption, i.e., $\mathcal{E}_{i, j}=0$. The status of the PN and SN BSs are set in two vectors denoted by $\epsilon_{p}$ and $\epsilon_{s}$, respectively.

3) Adopting Renewable Energy: In addition to the fossil fuel based energy, the cells need to be connected to a microgrid offering a green-generated energy, e.g., wind or solar energy. We denote by $\mathcal{E}_{i, j}^{(g)}$ the corresponding energy of the $j$-th BS in the network $i$. Hence, the cells are first powered by the available green energy, then are switched to the fossil fuel in the case where the green energy is insufficient.

4) Dynamic Energy Pricing: We assume that the price of fossil fuel energy varies with the BS consumption whereas the price of the green energy, denoted by $c^{(g)}$, is constant [15]. Hence, the energy cost function of the BS $j$ in the network $i$, denoted by $c_{i, j}$ is given by

$$
c_{i, j}=\left\{\begin{array}{cl}
c^{(g)}, & \text { if } \mathcal{E}_{i, j} \leq \mathcal{E}_{i, j}^{(g)} \\
c^{(g)}+f\left(\mathcal{E}_{i, j}\right), & \text { if } \mathcal{E}_{i, j}>\mathcal{E}_{i, j}^{(g)},
\end{array}\right.
$$

where $f($.$) is the price variation with the consumption.$ This model is related to the sensitivity of real-time energy demand to the fossil fuel.

\section{Dynamic Spectrum Management}

The available bandwidth $W$ is dynamically shared by the two networks with two spectrum management models:

- Spectrum leasing: The PN leases a fraction or the totality of the spectrum to the $\mathrm{SN}$ with a price $p_{s l}$. The fraction of leased bandwidth is denoted by $\beta_{p, j}$, $0 \leq \beta_{p, j} \leq 1$. From the SN side, we denote by $\beta_{s, j}$ the bandwidth fraction that the SN needs to be able to serve the users and we have $0 \leq \beta_{s, j} \leq \beta_{p, j}$.

- Spectrum sharing: The PN allows the SN to share a fraction of the bandwidth with a price $p_{s s}$ given by $\left(1-\beta_{p, j}\right)$. Meanwhile, the $\mathrm{SN}$ transmission is required to limit its interference to a given threshold denoted by $I_{\text {th }}$.

\section{iII. Profit Maximization and Constraints} FORMULATION

\section{A. Primary Profit Expression and Constraints}

The operational PN profit, denoted by $\Pi_{p}$, is defined as:

$$
\begin{aligned}
\Pi_{p} & =\sum_{j=1}^{N_{\mathrm{BS}}} \Pi_{p, j}=\underbrace{\left(\sum_{j=1}^{N_{\mathrm{BS}}} p_{p, \mathrm{op}}\left(N_{p, j}+N_{p, j}^{(\text {roamed })}\right)\right)}_{\text {Service profit }} \\
& +\underbrace{\sum_{j=1}^{N_{\mathrm{BS}}}\left(1-\epsilon_{p, j}\right)\left(p_{s l} \beta_{s, j} W-p_{\text {roam }} N_{p, j}^{\text {(roamed })}\right)}_{\text {Inactive BS profit }} \\
+ & \underbrace{\sum_{j=1}^{N_{\mathrm{BS}}} \epsilon_{p, j}\left(p_{s s}\left(1-\beta_{s, j}\right) W+p_{s l} \beta_{s, j} W-c_{p, j} \mathcal{E}_{p, j}\right)}_{\text {Active BS profit }},
\end{aligned}
$$

where $\Pi_{p, j}$ is the PN profit at the cell $j, p_{p, \text { op }}$ is the operation fees per PN user, $N_{p, j}^{\text {(roamed) }}$ is the count of all offloaded users in the cell $j$, and $p_{\text {roam }}$ is the roaming fees per user. The decision variables of the primary problem are $\epsilon_{p, j}, \beta_{p, j}, N_{p, j}^{\text {(roamed) }}$ for $j=1, \cdots, N_{\mathrm{BS}}$. Since $\beta_{s, j}$ is a variable of the secondary problem, we choose $\beta_{s, j}=$ $\beta_{p, j}$ while solving the primary problem, meaning that the $\mathrm{SN}$ is assumed to use all the available spectrum fraction offered by the PN. The PN profit maximization is subject to the following constraints

- Roamed users constraint: The roamed users are limited by the SN service capacity denoted by $\bar{N}_{s}$, i.e.,

$$
0 \leq N_{p, j}^{(\text {roamed })} \leq \bar{N}_{s} .
$$

- Service outage constraint: The PN needs to ensure good QoS by having a number of users in outage lower than an outage threshold denoted by $P_{\text {out }}$, i.e.,

$$
\frac{N_{p}-\sum_{j=1}^{N_{\mathrm{BS}}}\left(N_{p, j}+N_{p, j}^{\text {(roamed) }}\right)}{N_{p}} \leq P_{\text {out }} .
$$

However, this outage condition is not required by the $\mathrm{SN}$ since (i) the spectrum resource and $\mathrm{CO}_{2}$ footprint are mainly correlated to the PN operation (ii) having higher priority, the roamed $\mathrm{PN}$ users are served before the SN users benefiting from a besteffort service. 
- $\mathrm{CO}_{2}$ footprint constraint: The $\mathrm{PN} \mathrm{CO}_{2}$ emissions is below a threshold denoted by $C_{t h}^{P}$ defined as:

$$
\mathcal{F}_{p}^{\mathrm{CO}_{2}}=\sum_{j=1}^{N_{\mathrm{BS}}} \mathcal{F}_{p, j}^{\mathrm{CO}_{2}} \leq C_{t h}^{P}
$$

Note that the real threshold, denoted by $\overline{\mathrm{CO}_{2}}$ should not be exceeded by the sum of PN and SN emissions, i.e., $C_{t h}^{P}+C_{t h}^{S} \leq \overline{\mathrm{CO}_{2}}$ where $C_{t h}^{S}$ is the $\mathrm{SN}$ $\mathrm{CO}_{2}$ emissions threshold, where $\overline{\mathrm{CO}_{2}}=N_{\mathrm{BS}} \times \overline{\mathrm{CO}_{2}}$ and $\overline{\mathrm{CO}_{2}}$ is the $\mathrm{CO}_{2}$ emissions allowed in the area one cell.

\section{B. Secondary Profit Expression and Constraints}

The SN maximizes its profit when exploiting the leased or the shared spectrum by serving its users or collecting the roaming fees, respectively. The expression of the SN profit is

$$
\begin{aligned}
\Pi_{s}= & \underbrace{\sum_{j=1}^{N_{\mathrm{BS}}} \epsilon_{s, j} p_{s, \mathrm{op}} N_{s, j}}_{\text {Service profit }}-\underbrace{\sum_{j=1}^{N_{\mathrm{BS}}} \epsilon_{s, j} c_{s, j} \mathcal{E}_{s, j}}_{\text {Energy cost }} \\
& +\underbrace{\sum_{j=1}^{N_{\mathrm{BS}}} \epsilon_{s, j}\left(1-\epsilon_{p, j}\right)\left(p_{\text {roam }} N_{p, j}^{(\text {roamed })}-p_{s l} \beta_{s, j} W\right)}_{\text {Inactive PN BS profit }} \\
& -\underbrace{\sum_{j=1}^{N_{\mathrm{BS}}} \epsilon_{s, j} \epsilon_{p, j}\left(p_{s s}\left(1-\beta_{s, j}\right) W+p_{s l} \beta_{s, j} W\right)}_{\text {Active PN BS profit }},
\end{aligned}
$$

where $p_{s, \text { op }}$ is the operation fees per $\mathrm{SN}$ user.

The variables of the $\mathrm{SN}$ problem are $\epsilon_{s, j}, \beta_{s, j}, N_{s, j}$ for $j=1, \cdots, N_{\mathrm{BS}}$ which are subject five constraints:

- Power budget constraint: The SN transmit power, i.e., $\sum_{\sigma=1}^{\Sigma_{s}} N_{s, j} \bar{p}_{s, j}^{(\sigma)}+\sum_{\sigma=1}^{\Sigma_{p}} N_{p, j}^{(\text {roamed })} \bar{p}_{p \rightarrow s, j}^{(\sigma)}$ must be below the available power budget, denoted by $\bar{P}_{s}$, where $\bar{p}_{p \rightarrow s, j}^{(\sigma)}$ is the transmit power of the roamed users exploiting $\sigma$ in the $j$-th cell. This constraint can be written as:

$$
\sum_{\sigma=1}^{\Sigma_{s}} N_{s, j} \bar{p}_{s, j}^{(\sigma)}+\sum_{\sigma=1}^{\Sigma_{p}} N_{p, j}^{(\text {roamed })} \bar{p}_{p \rightarrow s, j}^{(\sigma)} \leq \bar{P}_{s} .
$$

- Interference constraint: The SN interference at the PN BS must be lower than a certain interference threshold $I_{\text {th }}$.

This constraint can be written as:

$$
\bar{p}_{s, j}^{(\sigma)} \frac{K}{\mathbb{E}_{p, \sigma}\left[d_{s, j}^{\nu}\right]} \leq I_{\mathrm{th}}, \forall j=1, \cdots, N_{\mathrm{BS}} .
$$

- Service capacity constraint: All served users, including the roamed PN ones, must be below the SN service capacity.
Hence, we have,

$$
N_{s, j} \leq \bar{N}_{s}-N_{p, j}^{\text {(roamed) }}, \forall j=1, \cdots, N_{\mathrm{BS}} .
$$

Note that in this constraint the variable to be optimized is $N_{s, j}$ whereas in (8) the variable is $N_{p, j}^{\text {(roamed) }}$

- $\mathbf{C O}_{2}$ footprint constraint: The $\mathrm{SN} \mathrm{CO}_{2}$ emissions is below a threshold as follows:

$$
\mathcal{F}_{s}^{\mathrm{CO}_{2}}=\sum_{j=1}^{N_{\mathrm{BS}}} \mathcal{F}_{s, j}^{\mathrm{CO}_{2}} \leq C_{t h}^{S} .
$$

- Spectrum leasing constraint: The SN can fully use a leased fraction of the bandwidth smaller than the leased fraction that the $\mathrm{PN}$ is offering

$$
0 \leq \beta_{s, j} \leq \beta_{p, j}, \forall j=1, \cdots, N_{\mathrm{BS}} .
$$

In the next section, we present an algorithm that solves the PN profit maximization problem as well as the $\mathrm{SN}$ profit maximization.

\section{Proposed Low-Complexity Algorithm}

In order to allow the $\mathrm{SN}$ to transmit while respecting the $\mathrm{CO}_{2}$ constraint, we assume that the $\mathrm{PN} \mathrm{CO}_{2}$ footprint is limited by a fraction of the total allowed emission and denoted by $\alpha$, i.e., $C_{t h}^{P}=\alpha \overline{\mathrm{CO}_{2}}$ and $C_{t h}^{S}=(1-\alpha) \overline{\mathrm{CO}_{2}}$. After maximizing its profit, the PN communicates the decided variables to the $\mathrm{SN}$ to maximize its profit given the $\mathrm{CO}_{2}$ emissions fraction $1-\alpha$.

\section{A. PN Profit Maximization}

We formulate the PN profit maximization problem as:

$$
\underset{\epsilon_{p, j}, \beta_{p, j}, N_{p, j}^{\text {(roamed })}}{\operatorname{maximize}} \Pi_{p}
$$

Subject to: (8), (9), (10),

$$
\epsilon_{p, j} \in\{0,1\}, 0 \leq \beta_{p, j} \leq 1,
$$

which is a combinatorial NP-Hard optimization problem where the optimal solution cannot be derived analytically and requires an exhaustive search (ES) method characterized by a high complexity. Instead, we propose a nearoptimal solution based on a low-complexity algorithm shown to have a close performance to those of the ES.

Our proposed algorithm is based on switching off the BSs that have low profit and high $\mathrm{CO}_{2}$ emissions. The way to decide which $\mathrm{BS}$ to be selected is to compute the profits and the $\mathrm{CO}_{2}$ emissions of all the PN BSs in the two status; active and inactive. Then, the parameters $\beta_{p, j}, N_{p, j}^{\text {(roamed) }}$ are derived, for $j=1, \ldots, N_{\mathrm{BS}}$, as:

- if $\epsilon_{p, j}=1$, then $\left\{\begin{array}{l}\beta_{p, j}=1-\sum_{\sigma}^{\Sigma_{p}} \frac{w_{p, j}^{(\sigma)} N_{p, j}^{\sigma}}{W}, \\ N_{p, j}^{\text {(roamed })}=0\end{array}\right.$

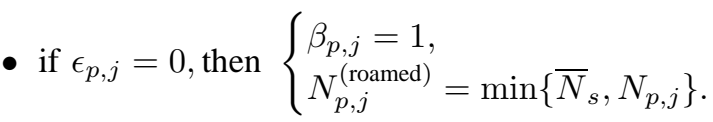


The next step is to switch off the BSs that have a higher profit when inactive than when they are active. This can be the case when the energy used to serve the PN users in a cell is higher than the roaming fees to be paid if they are roamed to the SN.

Hence, the obtained $\epsilon_{p}$ represents the solution if the outage constraint and the roamed users constraint are respected. Otherwise, more BSs are needed to be switched off until respecting all the constraints. For this reason, we introduce the ratio of the profit of a BS over its emission as a new metric that we call it profit per $\mathrm{CO}_{2}$ emissions $(\$ / K g)$. We, then, use this metric to switch off the BS with which the profit per $\mathrm{CO}_{2}$ emissions is the lowest among the other BSs. We summarize our proposed algorithm in Algorithm 1.

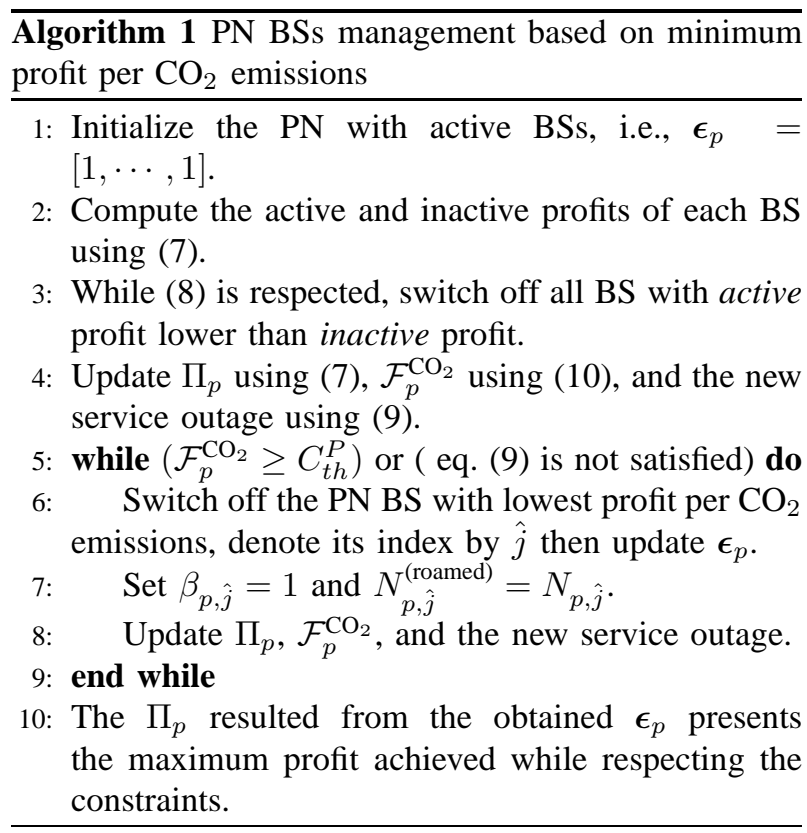

\section{B. SN Profit Maximization}

The SN profit maximization problem is defined as

$$
\underset{\epsilon_{s, j}, \beta_{s, j}, N_{s, j}}{\operatorname{maximize}} \Pi_{s}
$$

Subject to: (12), (13), (14), (15), (16).

The SN starts by determining the needed bandwidth fraction $\beta_{s, j}$ after serving the $\mathrm{PN}$ roamed users and having the values of $\boldsymbol{\epsilon}_{\boldsymbol{p}}$ and $\beta_{p, j}$ from the PN as part of the cooperation. In addition, $\beta_{s, j}$ is computed while respecting (3) and (13). If the $\mathrm{CO}_{2}$ constraint is not met by the SN, some SN BSs need to be switched off until having the $\mathrm{CO}_{2}$ emissions under $C_{t h}^{S}$. Note that the SN can only switch off the BS that corresponds to an active $\mathrm{PN}$ cell. Otherwise, the roamed PN users will not be served. Besides, the SN starts by switching off the BS having the lowest profit per $\mathrm{CO}_{2}$.

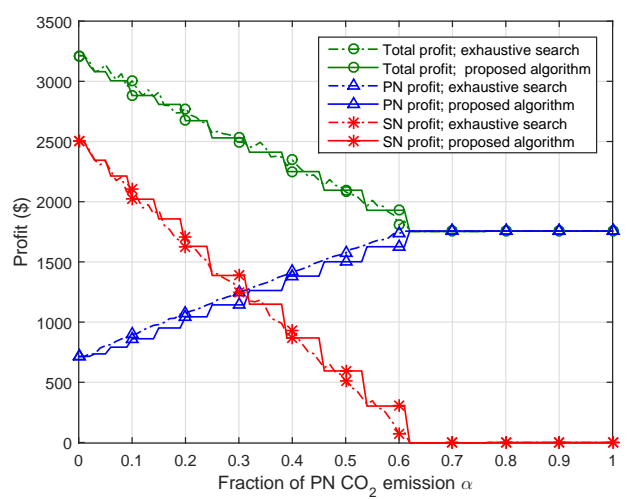

Fig. 2: $\mathrm{PN}$ and $\mathrm{SN}$ profits as function of the $\mathrm{CO}_{2}$ emissions fraction $\alpha$.

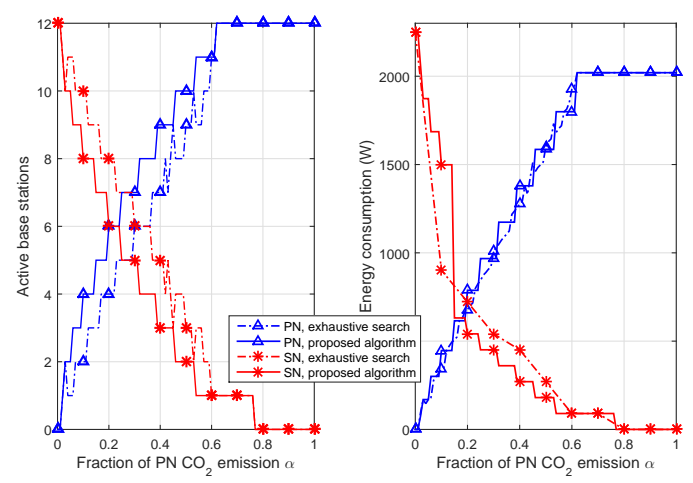

Fig. 3: PN and SN energy consumption and active BSs as function of the fraction of $\mathrm{CO}_{2}$ emission $\alpha$.

\section{Numerical Results}

We consider 12 BS deployed for the PN and the $\mathrm{SN}$, the adopted prices and parameters are listed in Table I [14]. The monetary unit is denoted by $\$$.

The PN and SN profits as well as their sum are plotted as a function of the fraction of $\mathrm{PN} \mathrm{CO}_{2}$ emissions, $\alpha$, in Fig. 2, for both the proposed algorithm and the ES. We show that PN profit of our proposed algorithm reaches values about $90 \%-100 \%$ of the ES profit. In the SN side, the profit corresponding to the proposed algorithm is higher than the ES profit allowing the sum of PN and $\mathrm{SN}$ profits to be almost the same. In addition, we notice that the PN profit corresponding to the proposed algorithm is piece-wise constant.

In order to understand this shape, we plot the PN active BSs and energy consumption as a function of $\alpha$ in Fig. 3. We notice that the proposed algorithm orders the PN BSs, given their profit per $\mathrm{CO}_{2}$ emissions. As $\alpha$ increases, the allowed $\mathrm{PN} \mathrm{CO}_{2}$ emissions is higher and the next BS in the ordering can be activated. This fact causes the piece-wise constant curve shapes reflected in the energy consumption. 
TABLE I: Adopted network prices and parameters

\begin{tabular}{|l|c||l|c|}
\hline Parameter & Value & Parameter & Value \\
\hline \hline Number of BSs $N_{B S}$ & 12 & Cell radius $(\mathrm{m})$ & 500 \\
\hline Total bandwidth $W(\mathrm{MHz})$ & 55 & Allowed outage $P_{\text {out }}$ & $5 \%$ \\
\hline Sharing price $p_{s s}(\$ / \mathrm{MHz})$ & 100 & Leasing price $p_{s l}(\$ / \mathrm{MHz})$ & 120 \\
\hline Received power $P_{\min }^{(\sigma)}(\mathrm{dBm})$ & -90 & Interf. threshold $I_{\text {th }}(\mathrm{dB})$ & 0 \\
\hline Pathloss constant $K(\mathrm{~dB})$ & -128.5 & Roaming price $p_{\text {roam }}(\$)$ & 4.25 \\
\hline Pathloss exponent $\nu$ & 3.76 & Rate per user $r_{i}^{(\sigma)}(\mathrm{kbps})$ & 250 \\
\hline Pollutant coefficients $\phi_{p}, \phi_{s}$ & 0.01 & Service prices $p_{\text {op } i}^{(\sigma)}(\$)$ & 4 \\
\hline Pollutant coefficients $\psi_{p}, \psi_{s}$ & 0.1 & Service Capacity $C_{i}$ & 50 \\
\hline $\mathrm{CO}_{2}$ limit per BS $C_{t h}^{P}(\mathrm{Kg})$ & 90.3 & BS constant power $b_{i}(W)$ & 71.5 \\
\hline $\mathrm{CO}_{2}$ emissions fraction $\alpha$ & 0.4 & Scaling parameter $a_{i}$ & 7.84 \\
\hline
\end{tabular}

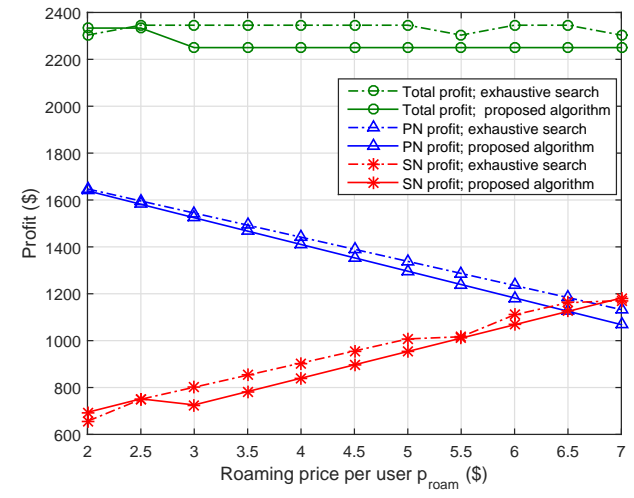

Fig. 4: PN and SN profits as function of the roaming price $p_{\text {roam }}$.

In Fig. 4, the PN and SN profits are plotted as a function of $p_{\text {roam. }}$. We show that both PN and SN variations are remarkable as the PN decreases and the SN increases with $p_{\text {roam }}$. In addition, both profits intersect in a specific value of $p_{\text {roam }}$, equal to $6.5 \$$ in our case, ensuring equal profits of both PN and SN networks. Hence, $p_{\text {roam }}$ is a key parameter that can regulate the market when needed. Hence, even if $p_{\text {roam }}$ is just $3-5 \%$ of the price of sharing and leasing, its change has a strong impact on the profit for the PN and the SN.

\section{CONCLUSION}

We proposed a cooperative spectrum and power allocation low-complexity algorithm for cognitive cellular networks. The objective is to maximize the profits of the primary and secondary networks (PN and $\mathrm{SN}$ ) under $\mathrm{CO}_{2}$ emissions constraints by turning off one or more base stations (BSs). Our low-complexity algorithm uses a new metric called profit per $\mathrm{CO}_{2}$ emissions and decides which BSs should be switched off. In our simulations, we show that our proposed algorithm performs well compared to the exhaustive search high-complexity method. In addition, the roaming price affects both profits and can be used to regulate the market.

\section{REFERENCES}

[1] L. Sboui, H. Ghazzai, Z. Rezki, and M.-S. Alouini, "On green cognitive radio cellular networks: Dynamic spectrum and operation management," IEEE Access, vol. 4, pp. 4046 - 4057, July 2016.

[2] G. Gur and F. Alagoz, "Green wireless communications via cognitive dimension: an overview," IEEE Network, vol. 25, no. 2, pp. 50-56, Mar. 2011.

[3] M. Erol-Kantarci and H. Mouftah, "Energy-efficient information and communication infrastructures in the smart grid: A survey on interactions and open issues," IEEE Communications Surveys Tutorials, vol. 17, no. 1, pp. 179-197, First Quarter, 2015.

[4] Ericsson, "ICT and the low carbon economy," Extract from the Ericsson Mobility Report, Nov. 2015.

[5] L. Sboui, Z. Rezki, and M.-S. Alouini, "Energy-efficient power allocation for underlay cognitive radio systems," IEEE Trans. on Cognitive Communications and Networking, vol. 1, no. 3, pp. 273-283, 2015.

[6] X. Hong, J. Wang, C.-X. Wang, and J. Shi, "Cognitive radio in 5G: a perspective on energy-spectral efficiency trade-off," IEEE Communications Magazine, vol. 52, no. 7, pp. 46-53, July 2014.

[7] L. Sboui, Z. Rezki, and M.-S. Alouini, "On energy efficient power allocation for power-constrained systems," in Proc. of IEEE PIMRC'14, Washington, DC, USA, Sep. 2014, pp. 19541958.

[8] L. Sboui, H. Ghazzai, Z. Rezki, and M.-S. Alouini, "Green collaboration in cognitive radio cellular networks with roaming and spectrum trading," in IEEE Symposium on Personal, Indoor and Mobile Radio Communications (PIMRC'15), Hong Kong, China, Aug. 2015.

[9] L. Sboui, H. Ghazzai, Z. Rezki, A. Sultan, and M.-S. Alouini, "Energy-efficient power allocation for UAV cognitive radio systems," in Proc. of the IEEE Vehicular Technology Conference (VTC'17F), Toronto, Canada, Sep. 2017.

[10] Y. Guo, J. Xu, L. Duan, and R. Zhang, "Joint energy and spectrum cooperation for cellular communication systems," IEEE Transactions on Communications, vol. 62, no. 10, pp. 36783691, Oct. 2014.

[11] A. Fehske, P. Marsch, and G. Fettweis, "Bit per joule efficiency of cooperating base stations in cellular networks," in IEEE GLOBECOM Workshops (GC Wkshps 2010), Miami, FL, USA, Dec. 2010.

[12] O. Arnold, F. Richter, G. Fettweis, and O. Blume, "Power consumption modeling of different base station types in heterogeneous cellular networks," in in Proc. of the Future Network \& Mobile Summit, Florence, Italy, June 2010.

[13] R. M. Corless, G. H. Gonnet, D. E. Hare, D. J. Jeffrey, and D. E. Knuth, "On the lambertw function," Advances in Computational mathematics, vol. 5, no. 1, pp. 329-359, 1996.

[14] H. Ghazzai, E. Yaacoub, M.-S. Alouini, and A. Abu-Dayya, "Optimized smart grid energy procurement for LTE networks using evolutionary algorithms," IEEE Transactions on Vehicular Technology, vol. 63, no. 9, pp. 4508-4519, Nov. 2014.

[15] Z. Fan, "A distributed demand response algorithm and its application to PHEV charging in smart grids," IEEE Transactions on Smart Grid, vol. 3, no. 3, pp. 1280-1290, Sept. 2012. 